\title{
METOdOLOGÍA DE GESTIÓN DE PROYECTOS PARA MEJORAR LA EJECUCIÓN DE PROYECTOS DE INFRAESTRUCTURA POR ADMINISTRACIÓN DIRECTA DE LOS GOBIERNOS LOCALES EN LA PROVINCIA JORGE BASADRE DE LA REGIÓN TACNA 2018.
}

\author{
METHODOLOGY OF PROJECT MANAGEMENT TO IMPROVE THE EXECUTION OF \\ INFRASTRUCTURE PROJECTS BY DIRECT ADMINISTRATION OF LOCAL GOVERNMENTS IN JORGE \\ BASADRE PROVINCE OF THE TACNA REGION 2018
}

Jean Carlo Villanueva Vargas ${ }^{1}$

Presentado: $18 / 08 / 2018$

Aceptado: $17 / 09 / 2018$

Publicado online:27/12/2018

\section{RESUMEN}

Validación de la Metodología de Gestión para mejorar la ejecución de proyectos de Infraestructura por Administración Directa de los Gobiernos Locales en la Provincia Jorge Basadre de la Tacna. El tipo de investigación es aplicada porque está orientada a la validación de una metodología de estándar Internacional de prestigio a nivel internacional y buscar una la mejora en la ejecución de proyectos de infraestructura bajo la modalidad de infraestructura en la Provincia Jorge Basadre del Departamento de Tacna. Se concluye con un nivel de confianza del 95\%, que el nivel de validez que el desarrollar una Metodología de Gestión se mejora la eficiencia en la ejecución de proyectos de Infraestructura por administración Directa de los Gobiernos Locales en la Provincia Jorge Basadre de la Tacna, respecto al Alcance, costo y tiempo, propuesto, es alto, por lo tanto, constituye una alternativa viable para la solución del problema de investigación. Se Validó el diseño de la Metodología de Gestión para mejorar la ejecución de proyectos de Infraestructura por Administración Directa de los Gobiernos Locales en la Provincia Jorge Basadre de la Tacna, basada en siete procesos adecuados a Gestión pública.

Palabras clave: Administración Directa, Áreas de Conocimiento, Gestión Proyecto, Infraestructura, Metodología, PMBOK, Procesos y Proyecto.

\footnotetext{
${ }^{1}$ Magister en Ingeniería Civil con mención en Gerencia de la Construcción.
} 


\begin{abstract}
Validation of the Management Methodology to improve the execution of Infrastructure projects by Direct Administration of Local Governments in the Jorge Basadre de la Tacna Province. The type of research is applied because it is oriented towards the validation of an international standard methodology of international prestige and to seek an improvement in the execution of infrastructure projects under the infrastructure modality in the Jorge Basadre Province of the Department of Tacna. It is concluded with a level of confidence of $95 \%$, that the level of validity that the development of a Management Methodology improves the efficiency in the execution of infrastructure projects by direct administration of the Local Governments in the Province Jorge Basadre of the Tacna, regarding the Scope, cost and time, proposed, is high, therefore, it constitutes a viable alternative for the solution of the research problem. The design of the Management Methodology was validated to improve the execution of Infrastructure projects by Direct Administration of Local Governments in the Jorge Basadre de la Tacna Province, based on seven processes appropriate to Public Management.
\end{abstract}

Keywords: Direct Administration, Knowledge Areas, Project Management, Infrastructure, Methodology, $P M B O K$, Processes and Project.

\title{
INTRODUCCIÓN
}

En la ejecución de los proyectos de Infraestructura los gobiernos locales enfrentan dificultades en sus diferentes etapas, dificultades producto de la experiencia de los profesionales disponibles, y no en base a la experiencia acumulada de la empresa en sus anteriores proyectos. En la presente investigación se propone mejorar el éxito de los proyectos de infraestructura por Administración Directa, el que permitirá cumplir con metas del Proyecto. Así mismo se ha establecido que para alcanzar el éxito de un proyecto se debe cumplir con el alcance, costo y tiempo, para lo que nos basaremos en los cinco grupos de procesos inicio, planificación, ejecución, seguimiento - control y cierre.

La investigación plantea como objetivos (1) Validar una Metodología de Gestión para mejorar la ejecución de proyectos de Infraestructura por Administración Directa de los Gobiernos Locales en la Provincia Jorge Basadre de Tacna, (2) Diagnosticar la situación actual sobre la ejecución de proyectos de infraestructura por Administración Directa de los Gobiernos Locales en la Provincia Jorge Basadre de la Región Tacna, con la finalidad de establecer la necesidad de la investigación, (3) Diseñar los procesos y procedimientos de la metodología de gestión para mejorar la ejecución de proyectos de Infraestructura por Administración Directa de los Gobiernos Locales en la Provincia Jorge Basadre de Tacna, (4) Definir la propuesta de metodología para la ejecución de proyectos de Infraestructura por Administración Directa de los Gobiernos Locales en la Provincia Jorge Basadre de Tacna y (5) Calificar el nivel de aceptación de los procesos y procedimiento de la metodología de gestión propuesta por los expertos especializados en la ejecución de proyectos bajo la modalidad de administración directa. 
En la región Tacna desde el 2007 a la fecha tiene un importante ingreso económico de canon y sobre canon producto por regalías mineras, específicamente de la Mina ubicada de Toquepala, distrito de llabaya del departamento de Tacna, dando impulso a la generación de proyectos de infraestructura, proyectos económicos, proyectos sociales entre otros. Las que en su mayoría son ejecutados a cargo de los gobiernos Locales bajo la modalidad de Administración Directa. En la provincia Jorge Basadre un porcentaje importante de obras por administración directa sobrepasan el tiempo de ejecución del mismo, observando un claro problema de tiempo. Se observa que no existe una metodología de gestión con las herramientas adecuadas desde la elaboración de expediente técnico, una planificación definida según el tipo de proyecto, un seguimiento y control durante la ejecución del proyecto.

Gómez (2016), presenta un Modelo de Gestión para sistematizar y ordenar el alineamiento y control de un proyecto de edificación alineado a los requerimientos de los profesionales y empresas del sector construcción. Hidalgo (2017 concluye en que un Sistema integral de Gestión permite gerenciar un proyecto de saneamiento. Salgado (2010) afirma para lograr que un proyecto sea exitoso, se debe hacer que se cumplan muchas variables de manera sinérgica. Farje (2011) refiere que el PMBOK ha permitido adoptar una metodología ordenada y estructurada para gerencia de proyectos. La Guía del $\mathrm{PMBOK}^{\circledR}$ contiene el estándar, reconocido a nivel mundial. Por estándar se entiende un documento formal que describe normas, métodos, procesos y prácticas establecidos. Al igual que en otras profesiones, el conocimiento contenido en este estándar evolucionó a partir de las buenas prácticas reconocidas de los profesionales dedicados a la dirección de proyectos que han contribuido a su desarrollo. Proporciona y promueve un vocabulario común para el uso y la aplicación de los conceptos de la dirección de proyectos dentro de la profesión de la dirección de proyectos.

\section{Objetivos}

A. Diagnosticar la situación actual de la ejecución de proyectos de infraestructura por Administración Directa de los Gobiernos Locales en la Provincia Jorge Basadre de la Región de Tacna, con la finalidad de establecer la necesidad de la investigación.

B. Diseñar los procesos y procedimientos de una metodología de gestión para mejorar la ejecución de proyectos de Infraestructura por Administración Directa de los Gobiernos Locales en la Provincia Jorge Basadre de Tacna.

C. Definir la propuesta de metodología para la ejecución de proyectos de Infraestructura por Administración Directa de los Gobiernos Locales en la Provincia Jorge Basadre de Tacna.

D. Calificar el nivel de aceptación de los procesos y procedimiento de la metodología de gestión propuesta por los expertos especializados en la ejecución de proyectos bajo la modalidad de Administración Directa.

\section{METODOLOGÍA}

El tipo de investigación es aplicada porque está orientada a la validación de una metodología de estándar Internacional de prestigio a nivel internacional y buscar la mejora en la ejecución de proyectos de infraestructura bajo la modalidad de infraestructura en la Provincia Jorge Basadre del Departamento de Tacna. La población de estudio está compuesta por los responsables de obras que laboraron en los Gobiernos Locales de llabaya, Locumba e Ite de la Provincia Jorge Basadre, Región Tacna con la experiencia en la ejecución de proyectos de ejecución presupuestaria directa. La muestra entrevistada está compuesta por 20 profesionales que fueron responsables de la ejecución de los proyectos de Infraestructura. El 
instrumento fue el cuestionario, que fue diseñado considerando la modalidad de preguntas, categorizadas según la finalidad de la investigación.

\section{RESULTADOS}

El $55 \%$ de los profesionales entrevistados tienen edades entre 30 y 45 años, el $25 \%$ tienen edades mayores a 45 años y la diferencia de $20 \%$ tienen edades menores a 30 años de edad. El $80 \%$ son Ingenieros Civiles, el $15 \%$ son Arquitectos y la diferencia de $5 \%$ corresponden a otras Especialidades. El $65 \%$ tienen una experiencia de 5 a 10 años, el $15 \%$ tiene una experiencia de 3 a 5 años, el 10\% tiene una experiencia mayor que 10 años y la diferencia de $10 \%$ corresponden a la experiencia menor de 3 años.

\section{Percepción sobre la ejecución de proyectos}

Ejecución de Proyectos de Infraestructura bajo la modalidad de Administración Directa: el $70 \%$ Opinan que fue mala, el $15 \%$ opina que fue Regular y la diferencia de $15 \%$ corresponden que esta fue buena.

Causa General de mayor problema: el $40 \%$ cree que fue por falta de un modelo de Gestión, el $20 \%$ cree que fue por falta de asignación de presupuesto, el $25 \%$ cree que fue por falta de otras causas, el $15 \%$ cree que fue por falta de compromiso de los involucrados, el $10 \%$ cree que fue por falta de capacidad profesional y la diferencia de $5 \%$ corresponden que esta fue por falta de expedientes técnicos adecuados.

Se ha contado con procesos y procedimientos basados en base a una metodología de gestión de proyectos con estándares internacionales: el $80 \%$ indica que no se contó y la diferencia de $20 \%$ corresponde a que si se contó.

Deficiencias en la etapa de INICIO Y PLANIFICACION, el 70\% indica que hubo falta y/o fue deficiente y la diferencia de $30 \%$ que no fue deficiente

Organigrama: el $70 \%$ indica que NO hubo Falta y/o deficiente Organigrama y el 30\% indica que SI Hubo.

Definición del Cronograma de Ejecución, el 75\% indica que SI hubo Falta

Definición del Presupuesto: el 65\% indica que SI hubo Falta y/o deficiente definición

Definición Comunicaciones, el 55\% indica que NO hubo Falta y/o deficiente Definición Comunicaciones

Riesgos del proyecto, el $60 \%$ indica que SI hubo Falta y/o deficiente Riesgos del proyecto.

EJECUCION, SEGUIMIENTO, CONTROL Y CIERRE, el 70\% indica que SI hubo Falta y/o deficiente Actas de Reuniones.

EJECUCION, SEGUIMIENTO, CONTROL Y CIERRE, el 70\% indica que SI hubo Falta y/o deficiente Plan de Trabajo y su desarrollo.

EJECUCION, SEGUIMIENTO, CONTROL Y CIERRE, específicamente considera que la Falta y/o deficiente Elaboración de Informes Mensuales, el 70\% indica que SI hubo Falta y/o deficiente Riesgos del proyecto.

EJECUCION, SEGUIMIENTO, CONTROL Y CIERRE, específicamente en que se considera la Falta y/o deficiente Seguimiento dela adquisición delos recursos, se obtuvo que el $60 \%$ indica que NO hubo Falta y/o deficiente Seguimiento dela adquisición de los recursos.

EJECUCION, SEGUIMIENTO, CONTROL Y CIERRE, específicamente en que se considera la Falta y/o deficiente proceso de Efectuar las adquisiciones, se obtuvo que el $60 \%$ indica que NO hubo Falta y/o deficiente Riesgos del proyecto.

EJECUCION, SEGUIMIENTO, CONTROL Y CIERRE, específicamente en que se considera la Falta 
y/o deficiente Solicitudes de Cambios (Adicionales y/o Deductivos) y Registro de Polémica, se obtuvo que el $70 \%$ indica que SI hubo Falta y/o deficiente Riesgos del proyecto y/o deficiente Solicitudes de Cambios (Adicionales y/o Deductivos) y Registro de Polémica.

EJECUCION, SEGUIMIENTO, CONTROL Y CIERRE, se obtuvo que el $65 \%$ indica que SI hubo Falta y/o deficiente Aprobación de cambios.

EJECUCION, SEGUIMIENTO, CONTROL Y CIERRE, considera que el $80 \%$ indica que SI hubo Falta y/o deficiente Lista de Problemas y/o restricciones del Proyecto

EJECUCION, SEGUIMIENTO, CONTROL Y CIERRE, se obtuvo que el $65 \%$ indica que NO hubo Falta y/o deficiente Control de las Adquisiciones

EJECUCION, SEGUIMIENTO, CONTROL Y CIERRE, se obtuvo que el $85 \%$ indica que SI hubo Falta y/o deficiente Elaboración y entrega del Informe Final (Cierre Técnico y Financiero).

Respecto a cuál es el modelo de Gestión con estándar internacional que más conoce, el $80 \%$ indica el PMBOK, el $15 \%$ el PRINCE2 y el $5 \%$ AACE.

\section{Sobre calidad de propuesta}

\section{Conclusiones}

Respecto a la propuesta de incluir o mejorar el proceso en la etapa INICIO Y PLANIFICACION para alcanzar el éxito del proyecto, se obtuvo que el $85 \%$ indica que SI debería de Desarrollar un Acta de Constitución e inicio y la Diferencia que corresponde al 15\% indica que NO debería de Desarrollar un Acta de Constitución e inicio.

Respecto a la propuesta de incluir o mejorar el proceso en la etapa INICIO Y PLANIFICACION para alcanzar el éxito del proyecto, se obtuvo que el $85 \%$ indica que SI debería de Desarrollar el Listado de los Interesados y la Diferencia que corresponde al $15 \%$ indica que NO debería de Desarrollar el Listado de los Interesados

En tal sentido SI debe de incluirse o mejorar el proceso en la etapa antes mencionados, reflejando el dominio del conocimiento.

Respecto a la propuesta de incluir o mejorar el proceso en la etapa INICIO Y PLANIFICACION para alcanzar el éxito del proyecto, se obtuvo que el $85 \%$ indica que SI debería de desarrollar la Elaboración Informe de Revisión del Expediente Técnico y la Diferencia que corresponde al $15 \%$ indica que NO debería desarrollar Elaboración Informe de Revisión del Expediente Técnico.

Respecto a la propuesta de incluir o mejorar el proceso en la etapa INICIO Y PLANIFICACION para alcanzar el éxito del proyecto, se obtuvo que el $80 \%$ indica que SI debería de desarrollar la Elaboración Informe de Estado Situacional del Proyecto y la Diferencia que corresponde al $20 \%$ indica que NO debería desarrollar Elaboración Informe de Estado Situacional del Proyecto.

Respecto a la propuesta de incluir o mejorar el proceso en la etapa INICIO Y PLANIFICACION para alcanzar el éxito del proyecto, se obtuvo que el $80 \%$ indica que NO debería de desarrollar la Elaboración del Enunciado del alcance y la Diferencia que corresponde al $20 \%$ indica que SI debería desarrollar Elaboración del Enunciado del alcance.

Respecto a la propuesta de incluir o mejorar el proceso en la etapa INICIO Y PLANIFICACION para alcanzar el éxito del proyecto, se obtuvo que el $70 \%$ indica que SI debería de desarrollar la Elaboración del EDT del Proyecto y la Diferencia que corresponde al 30\% indica que NO debería desarrollar la Elaboración del EDT del Proyecto.

Respecto a la propuesta de incluir o mejorar el proceso en la etapa INICIO Y PLANIFICACION para alcanzar el éxito del proyecto, se obtuvo que el $60 \%$ indica que NO debería de desarrollar la Elaboración del Organigrama del Proyecto y la Diferencia que corresponde al $40 \%$ indica 
que SI debería desarrollar la Elaboración del Organigrama del Proyecto.

Respecto a la propuesta de incluir o mejorar el proceso en la etapa INICIO Y PLANIFICACION para alcanzar el éxito del proyecto, se obtuvo que el 95\% indica que SI debería de Desarrollo del Cronograma de Ejecución y la Diferencia que corresponde al 5\% indica que NO debería Desarrollo del Cronograma de Ejecución.

Respecto a la propuesta de incluir o mejorar el proceso en la etapa INICIO Y PLANIFICACION para alcanzar el éxito del proyecto, se obtuvo que el $85 \%$ indica que SI debería de Desarrollo del Cronograma Presupuesto y la Diferencia que corresponde al $15 \%$ indica que NO debería Desarrollo del Cronograma Presupuesto.

Respecto a la propuesta de incluir o mejorar el proceso en la etapa INICIO Y PLANIFICACION para alcanzar el éxito del proyecto, se obtuvo que el $85 \%$ indica que SI debería de Desarrollo el Cronograma de Recursos Humanos y la Diferencia que corresponde al $15 \%$ indica que NO debería Desarrollo el Cronograma de Recursos Humanos.

Respecto a la propuesta de incluir o mejorar el proceso en la etapa INICIO Y PLANIFICACION para alcanzar el éxito del proyecto, se obtuvo que el $70 \%$ indica que SI debería de Desarrollar el Cronograma de Adquisiciones y la Diferencia que corresponde al $30 \%$ indica que NO debería Desarrollar el Cronograma de Adquisiciones.

39 Respecto a la propuesta de incluir o mejorar el proceso en la etapa INICIO Y PLANIFICACION para alcanzar el éxito del proyecto, se obtuvo que el $80 \%$ indica que $\mathrm{SI}$ debería de Desarrollo de la Matriz de Comunicaciones y la Diferencia que corresponde al $20 \%$ indica que NO debería Desarrollo de la Matriz de Comunicaciones.

Respecto a la propuesta de incluir o mejorar el proceso en la etapa INICIO Y PLANIFICACION para alcanzar el éxito del proyecto, se obtuvo que el $70 \%$ indica que NO debería de Desarrollo de la Gestión de la Calidad y la Diferencia que corresponde al 30\% indica que SI debería Desarrollo de la Gestión de la Calidad.

Respecto a la propuesta de incluir o mejorar el proceso en la etapa INICIO Y PLANIFICACION para alcanzar el éxito del proyecto, se obtuvo que el $70 \%$ indica que SI debería de Desarrollo de la Matriz de Riesgos del proyecto y la Diferencia que corresponde al 30\% indica que NO debería Desarrollo de la Matriz de Riesgos del proyecto

Respecto a la propuesta de incluir o mejorar el proceso en la etapa EJECUCION, MONITOREO, CONTROL Y CIERRE para alcanzar el éxito del proyecto, se obtuvo que el $70 \%$ indica que $\mathrm{SI}$ debería de Desarrollar de Actas de Reunión y la Diferencia que corresponde al 30\% indica que NO debería Desarrollar de Actas de Reunión.

Respecto a la propuesta de incluir o mejorar el proceso en la etapa EJECUCION, MONITOREO, CONTROL Y CIERRE para alcanzar el éxito del proyecto, se obtuvo que el $75 \%$ indica que SI debería la Aprobación de Informe de Revisión del expediente y Situacional de Obra y la Diferencia que corresponde al $25 \%$ indica que NO debería la Aprobación de Informe de Revisión del expediente y Situacional de Obra.

Respecto a la propuesta de incluir o mejorar el proceso en la etapa EJECUCION, MONITOREO, CONTROL Y CIERRE para alcanzar el éxito del proyecto, se obtuvo que el $80 \%$ indica que $\mathrm{SI}$ debería la Ejecución Monitoreo del Plan de Trabajo y la Diferencia que corresponde al $20 \%$ indica que NO debería la Ejecución Monitoreo del Plan de Trabajo.

Respecto a la propuesta de incluir o mejorar el proceso en la etapa EJECUCION, MONITOREO, CONTROL Y CIERRE para alcanzar el éxito del proyecto, se obtuvo que el $65 \%$ indica que SI debería la Elaboración de Informes Mensuales Ajustado a un modelo de Gestión Ejecución Monitoreo del Plan de Trabajo y la Diferencia que corresponde al 35\% indica que NO debería la Elaboración de Informes Mensuales Ajustado a un modelo de Gestión.

Respecto a la propuesta de incluir o mejorar el proceso en la etapa EJECUCION, MONITOREO, CONTROL Y CIERRE para alcanzar el éxito del proyecto, se obtuvo que el $70 \%$ indica que NO 
debería Monitoreo dela adquisición de los recursos y la Diferencia que corresponde al $30 \%$ indica que SI debería Monitoreo dela adquisición de los recursos.

Respecto a la propuesta de incluir o mejorar el proceso en la etapa EJECUCION, MONITOREO, CONTROL Y CIERRE para alcanzar el éxito del proyecto, se obtuvo que el $60 \%$ indica que $\mathrm{SI}$ debería Ejecución y Monitoreo de las adquisiciones y la Diferencia que corresponde al $40 \%$ indica que NO debería Ejecución y Monitoreo de las adquisiciones.

Respecto a la propuesta de incluir o mejorar el proceso en la etapa EJECUCION, MONITOREO, CONTROL Y CIERRE para alcanzar el éxito del proyecto, se obtuvo que el $70 \%$ indica que $\mathrm{SI}$ debería el Desarrollo de Solicitud y aprobación de Cambios (Adicionales y/o Deductivos) y la Diferencia que corresponde al 30\% indica que NO debería el Desarrollo de Solicitud y aprobación de Cambios (Adicionales y/o Deductivos).

Respecto a la propuesta de incluir o mejorar el proceso en la etapa EJECUCION, MONITOREO, CONTROL Y CIERRE para alcanzar el éxito del proyecto, se obtuvo que el $85 \%$ indica que SI debería el Desarrollo de Monitoreo y/o Control del Cronograma y la Diferencia que corresponde al 15\% indica que NO debería el Desarrollo de Monitoreo y/o Control del Cronograma.

Respecto a la propuesta de incluir o mejorar el proceso en la etapa EJECUCION, MONITOREO, CONTROL Y CIERRE para alcanzar el éxito del proyecto, se obtuvo que el $85 \%$ indica que SI debería el Desarrollo de Monitoreo y/o Control del Costo y la Diferencia que corresponde al $15 \%$ indica que NO debería el Desarrollo de Monitoreo y/o Control del Costo.

Respecto a la propuesta de incluir o mejorar el proceso en la etapa EJECUCION, MONITOREO, CONTROL Y CIERRE para alcanzar el éxito del proyecto, se obtuvo que el $65 \%$ indica que $\mathrm{SI}$ debería el Desarrollo de Monitoreo y/o Control de los Recursos y Adquisiciones y la Diferencia que corresponde al 35\% indica que NO debería el Desarrollo de Monitoreo y/o Control de los Recursos y Adquisiciones.

Respecto a la propuesta de incluir o mejorar el proceso en la etapa EJECUCION, MONITOREO, CONTROL Y CIERRE para alcanzar el éxito del proyecto, se obtuvo que el 95\% indica que SI debería el Elaboración y Aprobación del Informe Final (Cierre Técnico y Financiero) y la Diferencia que corresponde al $5 \%$ indica que NO debería el Elaboración y Aprobación del Informe Final (Cierre Técnico y Financiero).

Respecto a que, si Hace falta de una metodología de gestión de proyectos acorde a los estándares internacionales aplicada a la administración directa, se obtuvo que el $85 \%$ indica que SI hace falta una metodología de Gestión acorde a estándares internacionales y la Diferencia que corresponde al $15 \%$ indica que NO hace falta una metodología de Gestión acorde a estándares internacionales.

En la Tabla $N^{\circ} 54$ y Figura 54 Respecto a que, si Hace falta de una metodología de gestión de proyectos acorde a los estándares internacionales aplicada a la administración directa, se obtuvo que el $85 \%$ indica que el desarrollar una metodología de Gestión SI mejoraría la ejecución de Proyectos bajo la modalidad de Administración Directa y la Diferencia que corresponde al $15 \%$ indica que el desarrollar una metodología de Gestión NO mejoraría la ejecución de Proyectos bajo la modalidad de Administración Directa.

Respecto a que, Usaría y/o aplicaría un modelo de Gestión Anteriormente mencionado, se obtuvo que el $100 \%$ SI Usaría una metodología de Gestión que contemple todas las Procesos anteriormente mencionados.

\section{PROPUESTA DE METODOLOGÍA DE GESTIÓN}

Es una metodología que tiene la finalidad de adecuar las buenas practicas del PMBOK 2018, 
referidos a los procesos y procedimientos aplicados a la Ejecución de Proyectos de infraestructura en los Gobiernos Locales de la Provincia Jorge Basadre del departamento de Tacna. La propuesta de modelo se basa en gran medida en la metodología del Instituto de Gerencia de Proyectos - PMI. El modelo que se propone desarrolla de manera conceptual todos los grupos de procesos del ciclo de vida de los proyectos de infraestructura, pero para efectos de esta investigación se propone su aplicabilidad en el inicio, planificación, ejecución, seguimiento y control, cierre de los proyectos, desarrollando los conceptos y los formatos necesarios para elaborar el plan de gestión de un proyecto de infraestructura (Fig. 01)

Figura 01: Metodología de la Propuesta

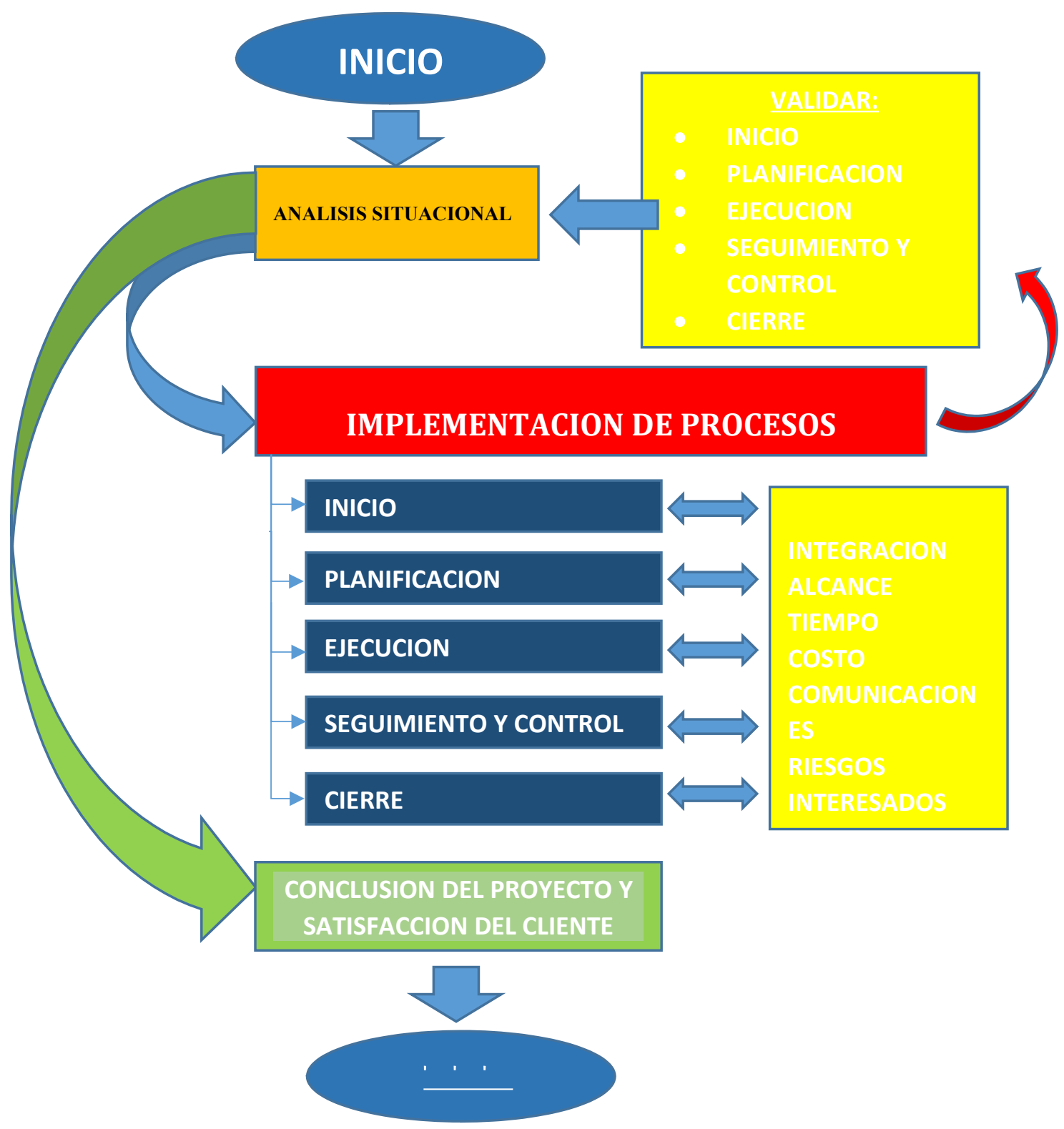

En la Figura 02 muestra la interrelación entre la gestión de proyectos por el modelo de gestión 
que está integrado que muestra los procesos, procedimientos y formatos estandarizados para proyectos de infraestructura. El PMBOK, como cuerpo de conocimiento es una guía de métodos, herramientas y técnicas agrupadas en áreas de conocimiento que en conjunto minimizan el riesgo de que un proyecto no alcance sus objetivos. El método o metodología para cada proyecto lo debe definir cada organización de acuerdo a diferentes intereses, productos, servicios, estructura, misión y objetivos organizacionales.

Figura 02: Esquema del Modelo de Gestión

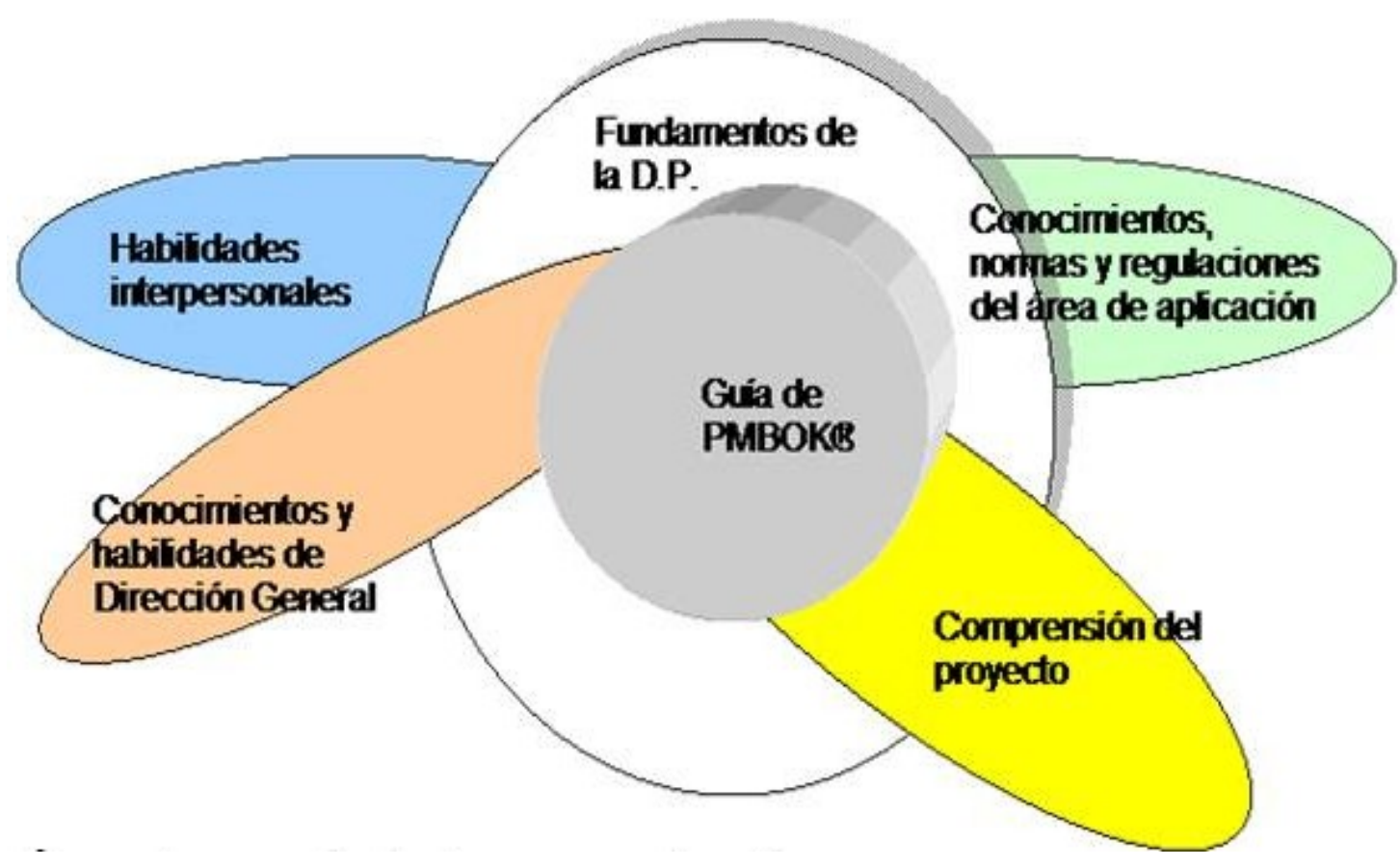

La Metodología de Gestión que se propone está estructurado acorde a los grupos de procesos del PMBOK, en los diferentes ciclos del proyecto, las que según el PMI son: inicio, planificación, ejecución, seguimiento y control y cierre. Cada uno de estos ciclos se explican y desarrolla con sus respectivos procesos para entender cuál es la forma de gestionar los proyectos de edificaciones en función de los procesos propuestos.

Se han desarrollado 17 formatos que son parte de los procesos de inicio, planificación, ejecución, seguimiento y control, estos formatos han sido desarrollados en función a los requerimientos específicos para la ejecución de proyectos de infraestructura bajo la modalidad de Administración Directa en os Gobiernos Locales de la Provincia Jorge Basadre del departamento de Tacna.

Se explican los procesos, procedimientos y formatos a seguir para la gestión de proyectos de infraestructura. En la presente investigación se desarrolla conceptualmente todos los procesos. 
Se desarrolla de manera clara, cada uno de los procesos, procedimientos en los formatos del modelo propuesto, Cada formato tienen un resumen y responde algunas preguntas sobre su utilización tales como ¿para qué sirven?, ¿que incluye?, ¿cuándo lo utilizarla? Además, se explicará según sea el caso, como cada uno de estos formatos, contribuye al desarrollo de los planes operativos al nivel de los operarios.

Tabla 01: DESCRIPCIÓN DE LOS RESULTADOS PREVISIBLES DE LA PROPUESTA

\begin{tabular}{|c|c|c|}
\hline $\begin{array}{l}\text { Procesos de la } \\
\text { Metodología }\end{array}$ & Nivel de logro validado por el experto & $\begin{array}{c}\text { Nivel de logro } \\
\text { esperado }\end{array}$ \\
\hline En el Inicio & $\begin{array}{l}\text { Gestión de Integración con la aplicación del Acta de constitución } \\
\text { para el inicio del Proyecto; Formato FG-01 }\end{array}$ & Alto Grado \\
\hline En el Inicio & $\begin{array}{l}\text { Gestión de Interesados con la Aplicación del Lista de Interesados; } \\
\text { Formato FG-02 }\end{array}$ & Alto Grado \\
\hline En la Ejecución & $\begin{array}{l}\text { Gestión de Alcance con la aplicación de la Declaración del Alcance; } \\
\text { Formato FG-03 }\end{array}$ & Alto Grado \\
\hline En la Ejecución & $\begin{array}{l}\text { Gestión de Alcance con la aplicación de la Estructura de Desglose } \\
\text { de Trabajo EDT; Formato FG-04 }\end{array}$ & Alto Grado \\
\hline En la Ejecución & $\begin{array}{l}\text { Gestión de la programación con la aplicación de la Matriz de } \\
\text { Funciones; Formato FG- } 05\end{array}$ & Alto Grado \\
\hline En la Ejecución & $\begin{array}{l}\text { Gestión de las comunicaciones con la aplicación del Calendario de } \\
\text { Eventos; Formato FG-06 }\end{array}$ & Alto Grado \\
\hline En la Ejecución & $\begin{array}{l}\text { Gestión de la programación con la aplicación del Estado semanal; } \\
\text { Formato FG-07 }\end{array}$ & Alto Grado \\
\hline En la Ejecución & $\begin{array}{l}\text { Gestión de la programación con la aplicación del Estado Mensual; } \\
\text { Formato FG-08 }\end{array}$ & Alto Grado \\
\hline En la Ejecución & $\begin{array}{l}\text { Gestión de la programación con la aplicación de la Programación } \\
\text { del Proyecto; Formato FG-09 }\end{array}$ & Alto Grado \\
\hline En la Ejecución & $\begin{array}{l}\text { Gestión de costos con la aplicación de la Estimación del Costo; } \\
\text { Formato FG-10 }\end{array}$ & Alto Grado \\
\hline En la Ejecución & $\begin{array}{l}\text { Gestión de costos con la aplicación del Cronograma Valorizado del } \\
\text { presupuesto; Formato FG-11 }\end{array}$ & Alto Grado \\
\hline En la Ejecución & $\begin{array}{l}\text { Gestión de riesgos con la aplicación de la Matriz de Riesgos del } \\
\text { Proyecto; Formato FG-12 }\end{array}$ & Alto Grado \\
\hline En la Ejecución & $\begin{array}{l}\text { Gestión de interesados con la aplicación de la Administración de } \\
\text { Cambios; Formato FG-13 }\end{array}$ & Alto Grado \\
\hline En la Ejecución & $\begin{array}{l}\text { Gestión de interesados con la aplicación de la Consejo } \\
\text { Descubiertos; Formato FG-14 }\end{array}$ & Alto Grado \\
\hline En la Ejecución & $\begin{array}{l}\text { Gestión de interesados con la aplicación de la Acta de Reuniones; } \\
\text { Formato FG-15 }\end{array}$ & Alto Grado \\
\hline Seguimiento y Control & $\begin{array}{l}\text { Gestión de integración con la aplicación de la Control del Avance; } \\
\text { Formato FG-16 }\end{array}$ & Alto Grado \\
\hline Cierre & $\begin{array}{l}\text { Gestión de integración con la aplicación de la Informe Final; } \\
\text { Formato FG-17 }\end{array}$ & Alto Grado \\
\hline
\end{tabular}

\section{DISCUSIÓN}

Se Validó la Metodología de Gestión para mejorar la efectividad en la ejecución de proyectos de Infraestructura por Administración Directa de los Gobiernos Locales en la Provincia Jorge Basadre de Tacna, basada en diecisiete procesos adecuados a la Gestión pública. El estado situacional diagnosticado permitió determinar que los profesionales encuestados que laboraron en la ejecución de proyectos de Infraestructura por Administración Directa de los Gobiernos Locales en la Provincia Jorge Basadre de Tacna, que tuvieron amplia experiencia; 
indicaron que la problemática respecto al mal estado situacional de la gestión de proyectos detectada era de urgente atención y sostienen que es necesario el diseño de una propuesta de metodología de gestión en los procesos de inicio, planificación, monitoreo y control y cierre de un proyecto de inversión pública en la etapa de ejecución. El diseño de los procesos procedimientos de la Metodología propuesta comprende diecisiete procesos que se deben de aplicar sistemáticamente en las Etapas de Inicio, Planificación, Control y Cierre en la ejecución de Proyectos de Infraestructura bajo la modalidad de Administración Directa, para asegurar la efectividad de los resultados. Consiste en formatos para los procesos de mayor necesidad o de problemática donde se incorporó Diagramas de Flujo, los que nos permiten observar el procedimiento teniendo en cuenta Tablas Resumen con las entradas, Herramientas de Trabajo y Salida, En siete Áreas de conocimiento como lo son la Gestión de la Integración, Gestión del alcance, Gestión de la Programación, Gestión de los Costos, Gestión de las Comunicaciones, Gestión de los Riesgos y la Gestión de los interesados. La metodología propuesta para mejorar la efectividad de la ejecución de proyectos de Infraestructura por Administración Directa de los Gobiernos Locales en la Provincia Jorge Basadre de Tacna, cumple su propósito en la medida que se implemente y desarrolle las acciones de cada proceso, de manera adecuada y pertinente. La validez del diseño de la Metodología propuesta se sostiene en lo confirmado con los expertos, que mejora la eficiencia en la ejecución de proyectos de Infraestructura por administración Directa de los Gobiernos Locales en la Provincia Jorge Basadre de Tacna, respecto al Alcance, costo y tiempo. En base a este trabajo se puede abrir futuras líneas de investigación en las diferentes etapas de ejecución de los proyectos de inversión pública.

\section{REFERENCIAS BIBLIOGRÁFICAS}

Alcina, J. (2006), "Como Cerrar Correctamente un Proyecto", Artículo, PMI, Venezuela.

Atkinson, R. (1999), "Project management: cost, time and quality, two best guesses and a phenomenon, its time to accept other success criteria", Artículo, International Journal of Project Management, USA.

Bititci, U.S. (1994), "Measuring your way to profit", Artículo, Management Accounting, USA.

Bresani, A (2009), "Gerencia de Operaciones", Articulo, U ESAN-MBA, Perú.

Briceño Balarezo (2003), "Implementación del Sistema de Planeamiento y Control de Costos por Procesos para Empresas de Construcción", Tesis UNMSM-EAPII, Perú.

Farje Mallqui, Julio Enrique (2011), “Aplicación de los lineamientos del PMBOK en la gestión de la ingeniería y construcción de un depósito de seguridad para residuos industriales", Tesis Perú.

Gomez Choquejahua, Omar Julinho (2016), “Modelo de gestión de proyectos de edificaciones para mejorar el planeamiento y control de la gestión de operaciones en la fase de ejecución", Tesis de Maestría en Ingeniería civil con mención en gerencia de construcción, Perú.

Hernan Porras, David (2013), "Gestión Administrativa de la construcción", Tesis de Maestría en Ingeniería civil, México DF.

Jaime B., Miguel C., Remigio C., Miguel R., Fernando T. (2002), GUÍA PARA UNA GESTIÓN BASADA EN PROCESOS -; Instituto Andaluz de Tecnología, imprenta Berekintza.

Hidalgo Aguilar, Omar Julinho (2017), "Desarrollo de un sistema integral de gestión (SIG) para mejorar la gestión de proyectos en obras de saneamiento en gobiernos locales", Tesis de Maestría en Ingeniería civil con mención en gerencia de construcción, Perú.

PMBOK 6th Edition (2018), A Guide to the Project Management Body of Knowledge.

Ley № 27972 (2013), "Ley Orgánica de Municipalidades”, Congreso de la República del Perú. 
Ley Na 30225 y D.S. N 350-2015-EF (2014), "Ley de Contrataciones del Estado y su Reglamento", Congreso de la República del Perú.

Salgado Canal, Jose (2010), "Importancia de la planificación, para el éxito de los proyectos, aplicando una metodología Estándar de gestión de proyectos"; Tesis Perú.

Salgado Canal, Jose (2013), "Implementación de una Metodología de Gestión en Proyectos Petroleros OffShore"; Congreso Internacional de Gestión de Proyectos, Project Management Institute 